\title{
REGIONAL DIFERENCES OF BREAST CANCER SURGERIES AMONG BRAZIL'S PUBLIC HELTHCARE USERS: A 10-YEAR STUDY
}

Jackeline R. O. Guidoux', Edésio Martins', Pollyana A. Gouveia1', Rosemar M. S. Rahal', Gustavo N. Guidoux', Ruffo Freitas-Junior ${ }^{1}$

'Breast Program, Teaching Hospital, Universidade Federal de Goiás - Goiânia (GO), Brazil.

Objective: Analyze if ocurred an increase of mastectomies and breast conservation surgery in patients treated by the Brazilian National Health System (Sistema Único de Saúde - SUS) between 2008 and 2017, as well as analyze if there were differences between the types of surgical procedure among Brazil's regions. Methodology: Descriptive time-series study of breast cancer surgeries, according to the population-based database on hospital information (DATASUS/SIH), all data of breast surgery were included, between 2008 and 2017. The absolute number of procedures was analyzed with Poisson regression, using Jointpoint Regression program and for the Annual Percentual Change (APC) was acquired for the trend, with a confidence interval of $95 \%$ and statistical significance when $\mathrm{p}<0.05$. A linearity test was used to assess the differences among Brazil's regions. Results: During the period studied, we found a decreasing trend of simple mastectomy in the South (APC -7.19) from 2008-2015; APC -53.87 from 2015-2018) and the Northeast (APC -14.34); in the North, we found a decreasing trend only during 2015-2017 (APC -42.52) and a stabilization trend in other regions. We only found an increasing trend of radical mastectomy in the North (APC 3.67); a decreasing trend was found in the Northeast (APC -3.5), in the Southeast (APC -2.56), and in the South (APC -1.66) Stabilization was only found in the Midwest. Relative to breast conservation surgery, the only region with an increased trend was the Midwest from 2008-2013 (APC 10.32); the South presented a decreasing trend (APC -5.13), Northeast from 2015-2017 (APC -29.61) and Midwest from 2013-2017 (APC -5.51), and stabilization was found in the North and the Southeast. The linearity test revealed that there is difference related to the type of surgery and regions, with statistical significance. Conclusions: During the period analyzed, we found a stabilization trend of breast surgeries in Brazil and in its regions. However, there were some differences among those regions, not only the total number of surgeries but also as the type of surgery performed. 